\title{
The interplay between cholesterol and breast cancer: is there a potential role for statin therapy?
}

\author{
Rahul Potluri*,1,2, Paul R Carter ${ }^{1,3}$, Deepthi Lavu ${ }^{1}$ \& Kevin R Bainey ${ }^{2}$ \\ ${ }^{1}$ ACALM Study Unit in collaboration with Aston Medical School, Aston University, Birmingham, UK \\ ${ }^{2}$ Mazankowski Alberta Heart Institute, University of Alberta, Edmonton, Alberta, Canada \\ ${ }^{3}$ Cambridge Epidemiology Unit, Worts' Causeway, University of Cambridge, Cambridge, UK \\ *Author for correspondence: rahulpotluri@outlook.com
}

"Gour understanding of the role of cholesterol and breast cancer is rapidly evolving and a number of recent observational studies suggest the need for a well-powered randomized clinical trial evaluating cholesterol lowering therapies (particularly statins) in patients with and/or at risk of developing breast cancer."’

First draft submitted: 28 February 2018; Accepted for publication: 5 March 2018; Published online: 27 July 2018

Keywords: breast $\bullet$ cancer $\bullet$ cholesterol $\bullet$ clinical $\bullet$ future $\bullet$ hyperlipidemia $\bullet$ prospects $\bullet$ review $\bullet$ statin $\bullet$ trial

High cholesterol has been established as a significant risk factor for cardiovascular disease and is well recognized by patients, clinicians, guideline committees and healthcare systems. A series of measures have been implemented to screen and treat high cholesterol largely by the use of statins and more recently by PCSK-9 inhibitors [1-4]. Over the last decade, evidence has emerged linking cholesterol with other modifiable risk factors such as obesity and diabetes [5,6]. Obesity has also been associated with the development of cancer. These associations have been echoed across a range of cancer types but particularly so in breast cancer [7-10]. Obesity and metabolic syndrome are known to be the most modifiable risk factors for the development of breast cancer in postmenopausal women [11].

In the late 20th century, some epidemiological studies (small sample sizes) made attempts to investigate the impact of serum cholesterol on the incidence of malignancy - however with inconclusive results [12,13]. Indirect evidence from dietary studies suggested that red and processed meats, which contain a high proportion of LDL cholesterol, are risk factors for colorectal, breast and endometrial cancer [14]. The field has evolved rapidly over the last 5 years, originating from a comprehensive study published in Science showing the role of cholesterol in the development of breast cancer in mice. Furthermore, the authors also showed that knock-out mice that developed high cholesterol and were treated with statins had a lower predisposition to developing breast cancer [15].

Subsequently, a large study in over 664,000 women utilizing Big Data from the UK Algorithm for Comorbidity, Associations, Length of Stay and Mortality (ACALM) registry found an association between diagnosis of hyperlipidemia and breast cancer [16]. This was far from conclusive given that selected previous epidemiological studies had demonstrated either no impact or an inverse relationship of high cholesterol on incidence of cancer [17,18]. Kitahara $e t$ al. investigated the role of cholesterol and associations with a number of cancers in a Korean registry of over 1 million patients and found high cholesterol to have a positive association with prostate, colon cancers in men and breast cancer in women but a negative association with liver and stomach cancer [19]. However, the complexity of investigating this relationship may become confounded by subtypes of cancer and mechanisms leading to high cholesterol (genetic vs environmental influences). Nevertheless, the potential for Big Data to help address this relationship between breast cancer and cholesterol has great value with incremental merit [16].

Upon reflection, biological plausibility becomes paramount to understand the complex interaction between cholesterol and breast cancer. At a cellular level, there are numerous physiologic mechanisms that may apply. Lowserum cholesterol may increase cell membrane fluidity, increasing the likelihood of neoplastic transformation [20]; alternatively, loss of membrane cholesterol may render tumor cells less antigenic, allowing transformed cells to escape

Future Medicine 
immune system surveillance [21]. Specifically in breast cancer, cholesterol was directly implicated in the development of estrogen receptor-positive breast cancer in mice via the metabolite 27-hydroxycholesterol (27HC) [15]. Malignancy growth was accelerated by increasing levels of $27 \mathrm{HC}$ and could be attenuated by administration of oral CYP27A1 inhibitors (the cytochrome P450 oxidase complex responsible for conversion to 27HC [15]. In human breast cancer specimens, these enzyme levels were seen to be more readily expressed in higher grade cancers could potentially have a greater effect [15].

In 2016, another large study showed that in patients with breast, prostate, lung and colorectal cancer, the presence of hyperlipidemia significantly improved their long-term mortality compared with cancer patients without hyperlipidemia [22]. However, the effect of lipid-lowering therapy in cancer has been unclear but widely discussed in the medical and lay press [23]. While the role of cholesterol lowering therapies having an impact in cancer patients has gained traction, earlier studies have shown little impact between the association of statin use and incidence of breast cancer [24]. Still, recent studies have demonstrated that statin-therapy started after diagnosis of malignancy in postmenopausal women improves survival [25]. In Demark, a prospective cohort study following 18,769 patients with limited-stage breast cancer during 1996-2003 showed that postdiagnosis use of the more lipophilic statins such as simvastatin were associated with reduced cancer recurrence rate in comparison with both those not taking a statin and those taking a hydrophilic statin such as pravastatin [26]. Simvastatin patients experienced approximately ten fewer breast cancer recurrences per 100 women after 10 years of follow-up compared with women who either were not prescribed a statin or on a hydrophilic statin [26]. This study provided further support for an earlier smaller study which found reduced recurrence rates after postdiagnosis use of a lipophilic statin and decreased recurrence rate in breast cancer [27]. The relationship between breast cancer and cholesterol is complex not only owing to variations in type and timing of diagnosis and therapy but also because of the fundamental question of whether high cholesterol itself prevents breast cancer or improves survival after diagnosis of breast cancer. Adding to the complexity is the interplay with statin therapy. This issue conceivably explains why initial literature on the subject report conflicting results [12-19].

This lead to the most recent and perhaps most comprehensive study on the topic answering both the relationship between high cholesterol and breast cancer development and long-term outcomes from the UK ACALM Big Data registry [28]. Over 16,000 hyperlipidemia patients without cancer were compared with a matched sample of nonhyperlipidemia patients without cancer and outcomes of breast cancer rates along with mortality were recorded over a 14-year period. Hyperlipidemia patients were approximately half as likely to develop breast cancer and those that developed breast cancer had $40 \%$ reduced mortality and improved long-term survival compared with nonhyperlipidemia patients [28]. Statins are the mainstay of treatment for hyperlipidemia patients in the UK according to a latest study on over 7 million patients, suggesting a potential role of statins in reducing breast cancer development and/or treatment [29]. Still, this interaction needs to be explored.

In spite of all the evidence to date regarding breast cancer, cholesterol and statins, it would be misleading to suggest that statins (or even latest generation lipid lowering therapies such as PCSK9 inhibitors) prevent or treat breast cancer. As such, it is imperative to explore a randomized clinical trial to help address this important clinical conundrum. The early statin trials exploring cardiovascular outcomes were inconclusive to explore the association with cancer (low number of cancer patients) [1,2]. Nowadays, statins are generic and the appetite for pharmaceutical company funded trials on the subject may be of diminishing return. Furthermore, the expenses will be compounded by the fact that a large number of patients would be required to be followed for an extended period of time in order to have the statistical power to ascertain treatment effect. Given the commonly recognized side effects of statins such as myalgia, myopathy and liver toxicity, the compliance among healthy patients may become an issue even though the accumulation of safety data on statins is likely to pass the most rigorous of ethical approvals [30].

Upon further reflection, an alternate strategy may be to look at the role of statins in patients who develop breast cancer. The number of patients for such a study is likely to be lower and may be more cost effective particularly if considering a large-scale randomized registry approach utilizing Big Data [31,32]. The main issue with such an approach would be noise from cardiovascular disease (CVD) outcomes and mortality. Over $50 \%$ of worldwide mortality is from CVD and/or cancer and even higher in western societies. There is considerable overlap with CVD being common in cancer patients and the European Society of Cardiology has even launched a series of guidelines highlighting these issues [33]. Statin treatment in such a scenario will protect from CVD which may translate into better outcomes in the treatment group. Regardless, if all-cause mortality is the outcome studied, this limitation is not clinically relevant as ultimately the goal is to improve treatment of breast cancer. 
In summary, our understanding of the role of cholesterol and breast cancer is rapidly evolving and a number of recent observational studies suggest the need for a well-powered randomized clinical trial evaluating cholesterol lowering therapies (particularly statins) in patients with and/or at risk of developing breast cancer.

\section{Financial \& competing interests disclosure}

The authors have no relevant affiliations or financial involvement with any organization or entity with a financial interest in or financial conflict with the subject matter or materials discussed in the manuscript. This includes employment, consultancies, honoraria, stock ownership or options, expert testimony, grants or patents received or pending, or royalties.

No writing assistance was utilized in the production of this manuscript.

\section{References}

Papers of special note have been highlighted as: • of interest

1. Scandinavian Simvastatin Survival Study Group. Randomized trial of cholesterol lowering in 4444 patients with coronary heart disease: the Scandinavian Simvastatin Survival Study (4S). Lancet 344(8934), 1383-1389 (1994).

- Original clinical trial showing benefit of statins in hyperlipidemia

2. Sever PS, Dahlö B, Poulter NR et al. Prevention of coronary and stroke events with atorvastatin in hypertensive patients who have average or lower-than-average cholesterol concentrations, in the Anglo-Scandinavian cardiac outcomes trial lipid lowering arm (ASCOT-LLA): a multicenter randomized controlled trial. Lancet 361(9364), 1149-1158 (2003).

3. Piepoli MF, Hoes AW, Agewall S et al. 2016 European guidelines on cardiovascular disease prevention in clinical practice: the Sixth Joint Task Force of the European Society of Cardiology and Other Societies on cardiovascular disease prevention in clinical practice (constituted by representatives of 10 societies and by invited experts) developed with the special contribution of the European Association for Cardiovascular Prevention and Rehabilitation (EACPR). Eur. Heart J. 37(29), 2315-2381 (2016).

4. Sabatine MS, Giugliano RP, Keech AC et al. Evolocumab and clinical outcomes in patients with cardiovascular disease. N. Engl. J. Med. 376(18), 1713-1722 (2017).

- $\quad$ Latest clinical trial showing PCSK9 inhibitors evidence in hyperlipidemia.

5. Kratz M, Baars T, Guyenet S. The relationship between high-fat dairy consumption and obesity, cardiovascular and metabolic disease. Eur. J. Nutr. 52(1), 1-24 (2013).

6. Onat A, Hergenc G, Sari I, Karabulut A, Can G. Elevated LDL-cholesterol level predicts diabetes in centrally obese women but not men: relative roles of insulin resistance and central obesity. Circ. J. 71(9), 1463-1467 (2007).

7. Berger NA. Obesity and cancer pathogenesis. Ann. NY Acad. Sci. 1311, 57-76 (2014).

- Comprehensive discussion of obesity and link to cancer.

8. Dubois V, Delort L, Billard H, Vasson MP, Caldefie-Chezet F. Breast cancer and obesity: in vitro interferences between adipokines and proangiogenic features and/or antitumor therapies. PLOS ONE 8(3), e58541 (2013).

9. De Pergola G, Silvestris F. Obesity as a major risk factor for cancer. J. Obes. 2013, 291546 (2013).

10. Protani M, Coory M, Martin JH. Effect of obesity on survival of women with breast cancer: systematic review and meta-analysis. Breast Cancer Res. Treat. 123(3), 627-35 (2010).

11. Neuhouser ML, Aragaki AK, Prentice RL et al. Overweight, obesity, and postmenopausal invasive breast cancer risk: a secondary analysis of the women's health initiative randomized clinical trials. JAMA Oncol. 1(5), 611-621 (2015).

12. Salonen JT. Risk of cancer and death in relation to serum cholesterol. a longitudinal study in an eastern Finnish population with high overall cholesterol level. Am. J. Epidemiol. 116(4), 622-630 (1982).

13. Wald NJ, Thompson SG, Law MR, Densem JW, Bailey A. Serum cholesterol and subsequent risk of cancer: results from the BUPA study. Br. J. Cancer 59(6), 936-938 (1989).

14. Guo J, Wei W, Zhan L. Red and processed meat intake and risk of breast cancer: a meta-analysis of prospective studies. Breast Cancer Res. Treat. 151(1), 191-198 (2015).

- Indirect evidence showing link between breast cancer and cholesterol.

15. Nelson ER, Wardell SE, Jasper JS et al. 27-Hydroxycholesterol links hypercholesterolemia and breast cancer pathophysiology. Science 342(6162), 1094-1098 (2013).

- Very important article showing mechanism of high cholesterol and breast cancer pathogenesis.

16. Potluri R, Lavu D, Uppal H, Chandran S. Hyperlipidemia as a risk factor for breast cancer? Cardiovasc. Res. 103S1, 135 (2014).

- Largest study showing association between cholesterol and breast cancer in humans.

17. Berglund G, Nilsson P, Eriksson KF et al. Long-term outcome of the Malmö preventive project: mortality and cardiovascular morbidity. J. Intern. Med. 247(1), 19-29 (2000).

18. Bahl M, Ennis M, Tannock IF et al. Serum lipids and outcome of early stage breast cancer: results of a prospective cohort study. Breast Cancer Res. Treat. 94(2), 135-144 (2005). 
19. Kitahara CM, Berrington de González A, Freedman ND et al. Total cholesterol and cancer risk in a large prospective study in Korea. J. Clin. Oncol. 29(12), 1592-1598 (2011).

20. Oliver MF. Serum cholesterol: the knave of hearts or the joker. Lancet 2, 1090-1095 (1981).

21. Shinitzky M. Membrane fluidity in malignancy: adversative and recuperative. Biochim. Biophys. Acta 738, 251-261 (1984).

22. Carter PR, McGowan J, Uppal H, Chandran S, Sarma J, Potluri R. Hyperlipidemia reduces mortality in breast, prostate, lung and bowel cancer. Heart 102, A57-A58 (2016).

23. Park A. Why statins could be the next treatment for breast cancer? Time 5th July 2014. http://time.com/2954609/statins-breast-cancer/

24. Undela K, Srikanth V, Bansal D. Statin use and risk of breast cancer: a meta-analysis of observational studies. Breast Cancer Res. Treat. 135(1), 261-269 (2012).

25. Nielsen SF, Nordestgaard BG, Bojesen SE. Statin use and reduced cancer-related mortality. N. Engl. J. Med. 367, 1792 (2012).

26. Ahern TP, Pedersen L, Tarp M et al. Statin prescriptions and breast cancer recurrence risk: a Danish nationwide prospective cohort study. J. Natl Cancer Inst. 103(19), 1461-1468 (2011).

27. Kwan ML, Habel LA, Flick ED et al. Postdiagnosis statin use and breast cancer recurrence in a prospective cohort study of early stage breast cancer survivors. Breast Cancer Res. Treat. 109(3), 573-579 (2008).

- Important study showing some evidence for statin and breast cancer.

28. Carter PR, Uppal H, Chandran S, Bainey KR, Potluri R. Patients with a diagnosis of hyperlipidemia have a reduced risk of developing breast cancer and lower mortality rates: a large retrospective longitudinal cohort study from the UK ACALM registry. Eur. Heart J. 38(1), 3106 (2017).

- Naturalistic follow-up study showing evidence between hyperlipidemia, breast cancer, follow-up and mortality.

29. O'Keeffe AG, Nazareth I, Petersen I. Time trends in the prescription of statins for the primary prevention of cardiovascular disease in the United Kingdom: a cohort study using the health improvement network primary care data. Clin. Epidemiol. 8, 123-132 (2016).

30. Collins R, Reith C, Emberson J et al. Interpretation of the evidence for the efficacy and safety of statin therapy. Lancet 388(10059), 2532-2561 (2016)

31. Fröbert O, Lagerqvist B, Gudnason T et al. Thrombus aspiration in ST-elevation myocardial infarction in Scandinavia (TASTE trial). A multicenter, prospective, randomized, controlled clinical registry trial based on the Swedish Angiography and Angioplasty Registry (SCAAR) platform: study design and rationale. Am. Heart J. 160(6), 1042-1048 (2010).

32. Hernandez AF, Fleurence RL, Rothman RL. The ADAPTABLE trial and PCORnet: shining light on a new research paradigm. Ann. Intern. Med. 163(8), 635-636 (2015).

33. Zamorano JL, Lancellotti P, Rodriguez Muñoz D et al. 2016 ESC position paper on cancer treatments and cardiovascular toxicity developed under the auspices of the ESC Committee for practice guidelines: the task force for cancer treatments and cardiovascular toxicity of the European Society of Cardiology (ESC). Eur. Heart J. 37(36), 2768-2801 (2016). 